\title{
Altered Functional Connectivity in Children with ADHD Revealed by Scalp EEG: An ERP Study
}

\author{
Chunli Chen, ${ }^{1}$ Huan Yang, ${ }^{2,3,4,5,6}$ Yasong Du, ${ }^{7}$ Guangzhi Zhai, ${ }^{8}$ Hesheng Xiong, \\ Dezhong Yao, ${ }^{1}$ Peng Xu $\mathbb{D}^{1},{ }^{1}$ Jianhua Gong $\mathbb{D}^{9},{ }^{9}$ Gang Yin $\mathbb{D}^{10},{ }^{10}$ and Fali Li ${ }^{1}{ }^{1}$ \\ ${ }^{1}$ School of Life Science and Technology, Center for Information in Medicine, University of Electronic Science and Technology of China, \\ Chengdu 611731, China \\ ${ }^{2}$ China National Clinical Research Center on Mental Disorders (Xiangya), Changsha 410011, China \\ ${ }^{3}$ China National Technology Institute on Mental Disorders, Changsha 410011, China \\ ${ }^{4}$ Hunan Technology Institute of Psychiatry, Changsha 410011, China \\ ${ }^{5}$ Hunan Key Laboratory of Psychiatry and Mental Health, Changsha 410011, China \\ ${ }^{6}$ Mental Health Institute of Central South University, Changsha 410011, China \\ ${ }^{7}$ Mental Health Center Affiliated to Medical School of Shanghai Jiao Tong University, 200030, China \\ ${ }^{8}$ Shenzhen Nao Qianneng Co., Ltd., 518002, China \\ ${ }^{9}$ Luohu District Maternity and Child Healthcare Hospital, Shenzhen 518019, China \\ ${ }^{10}$ Sichuan Cancer Hospital, School of Medicine, University of Electronic Science and Technology of China, Chengdu 610041, China
}

Correspondence should be addressed to Jianhua Gong; 768186112@qq.com, Gang Yin; cxqyguestc@163.com, and Fali Li; fali.li@uestc.edu.cn

Received 5 December 2020; Accepted 28 April 2021; Published 12 May 2021

Academic Editor: Jianzhong Su

Copyright (c) 2021 Chunli Chen et al. This is an open access article distributed under the Creative Commons Attribution License, which permits unrestricted use, distribution, and reproduction in any medium, provided the original work is properly cited.

\begin{abstract}
Attention deficit hyperactivity disorder (ADHD) is one of the most common neurodevelopmental brain disorders in childhood. Despite extensive researches, the neurobiological mechanism underlying ADHD is still left unveiled. Since the deficit functions, such as attention, have been demonstrated in ADHD, in our present study, based on the oddball P3 task, the corresponding electroencephalogram (EEG) of both healthy controls (HCs) and ADHD children was first collected. And we then not only focused on the event-related potential (ERP) evoked during tasks but also investigated related brain networks. Although an insignificant difference in behavior was found between the HCs and ADHD children, significant electrophysiological differences were found in both ERPs and brain networks. In detail, the dysfunctional attention occurred during the early stage of the designed task; as compared to HCs, the reduced P2 and N2 amplitudes in ADHD children were found, and the atypical information interaction might further underpin such a deficit. On the one hand, when investigating the cortical activity, HCs recruited much stronger brain activity mainly in the temporal and frontal regions, compared to ADHD children; on the other hand, the brain network showed atypical enhanced long-range connectivity between the frontal and occipital lobes but attenuated connectivity among frontal, parietal, and temporal lobes in ADHD children. We hope that the findings in this study may be instructive for the understanding of cognitive processing in children with ADHD.
\end{abstract}

\section{Introduction}

Attention deficit hyperactivity disorder (ADHD) is one of the most common childhood psychiatric disorders, characterized by age-inappropriate symptoms of inattention, hyperactivity, and impulsivity [1]. It arises in childhood and often persists into adolescence, even adulthood. The reduced-capacity [2] or dysfunctional attention [3] was not the major cause of ADHD symptoms; event-related potential (ERP), such as P2, N2, and P3, has consistently suggested the cognitive deficits in multiple stages of sensory and cognitive processing in ADHD [4]. P2, a positive deflection peaking around $150-250 \mathrm{~ms}$ after stimuli presentation, plays a crucial role when measuring attention [5], 
which could reflect not only early comprehension but also index updating of a representation in response to new incoming information [6]. A smaller P2 in ADHD reflects an underactivation of the early orienting process and a poor allocation of attentional resources [7]. Besides, N2, peaking at around $200-250 \mathrm{~ms}$ after stimuli onset, could reflect the cognitive control for successful inhibitory control and interference suppression [8] and has also been found to be reduced in ADHD patients [9]. P3 is a later ERP component and peaks around $250-600 \mathrm{~ms}$ and is widely used to investigate cognitive mechanisms in neuropsychiatric disorders, including ADHD [10]. For example, $\mathrm{Li}$ et al. suggested that the rest and task P3 electroencephalogram (EEG) could actually provide comprehensive information to reliably classify schizophrenia patients from healthy controls (HCs) [11]. Additionally, its amplitude has also been found to be decreased in ADHD [12]. As illustrated, the early stage may influence subsequent response processes [13]; since P2, N2, and P3 index different stages of cognitive information processing, in this work, all of these components were investigated to uncover the cognitive deficits in ADHD children.

The brain is not only a dynamic system but also a complex network [14]. Of note, other than isolated brain regions, the brain networks could reveal the information exchange and propagation among various brain regions [15] and provide more information to shed light on the relationship between cognition and the related brain network [16, 17]. Deficits of certain regions disturb the information processing in the brain and consequently lead to network failure [18]. ADHD was described as a network disorder [19], as ADHD has been demonstrated to be linked not only with the structural deficits of specific regions but also with the structural interconnectivity [20]. Structural magnetic resonance imaging studies have reported abnormal volume and cortical thickness in multiple brain regions [21], including prefrontal [22], temporal, and parietal [23] cortices, in ADHD. Network neuroscience [24] thus raised a "bridge" between ADHD and brain network analysis [25]. For example, Furlong et al. found that the increased global network efficiency is associated with elevated ADHD symptom severity [26]. Srinivasan suggested a static state of deficient connectivity in ADHD and a stimulus-induced state of overconnectivity within and between frontal hemispheres [27]. To understand the network mechanism underlying ADHD, we subsequently utilized the brain network analysis to probe the stimulus-induced network structure of ADHD children.

In this study, we hypothesized that the differences in ERP components could reflect on which stage the cognitive deficits in ADHD occurred, and brain network analysis might further uncover the potential network mechanism underlying the task behaviors of ADHD during the designed P3 tasks. Therefore, to better understand how ADHD children recruit cognitive resources to complete the tasks, the ERP and complex network analyses were adopted to investigate the potential differences between HCs and ADHD children.

\section{Materials and Methods}

2.1. Participants. A total of 71 children, including 40 ADHD children (35 males; mean age $=7.65$ years, standard deviation $(\mathrm{SD})=2.11$ years $)$ and $31 \mathrm{HCs}(20$ males; mean age $=7.68$ years, $\mathrm{SD}=2.36$ years) were recruited from Shenzhen Luohu District Maternity and Child Healthcare Hospital, China. ADHD children needed to meet the Diagnostic and Statistical Manual of Mental Disorders fifth edition (DSM5) criteria of ADHD diagnosis. Two psychiatrists made these diagnoses independently based on DSM-5 and needed to assess the children in two or more situations. For the control group, age-matched children did not have ADHD or any other psychiatric disorders. Exclusion criteria were neurological disorder, a history of brain injury, mental retardation, comorbid current psychiatric diagnosis, and other neurodevelopmental disorders.

2.2. Experimental Procedures. Our experiment complied with a standard oddball paradigm, as shown in Figure 1. The P3 task designed contained 30 target trials that needed the corresponding responses from subjects, along with 120 standard stimuli that did not require the subjects' responses, and during the whole P3 task, the stimuli were randomly presented. Herein, the standard stimulus was defined as the upward-oriented triangle with a thin cross in its center, while the target stimulus was defined as the down-oriented triangle with a thin cross in its center. In each trial, a $250 \mathrm{~ms}$ attention alert, a $500 \mathrm{~ms}$ preparation cue, a $500 \mathrm{~ms}$ stimulus presentation, and a $1000 \mathrm{~ms}$ break were included. Subjects were required to press the "1" key on a standard keyboard as quickly as possible once the target stimulus was presented.

2.3. EEG Data Acquisition. The EEG data were recorded with a 16-channel Ag/AgCl (i.e., Fp1/2, F3/4, C3/4, P3/4, O1/2, F7/8, T3/4, and T5/6) electrode cap (BrainMaster, Inc., Shenzhen, China). All electrodes were positioned according to the 10-20 international system. During online data recording, electrode $\mathrm{AFz}$ served as the reference. EEG signals were sampled at $1000 \mathrm{~Hz}$ and online bandpass filtered at 0.05$100 \mathrm{~Hz}$. During recording, the impedances of all electrodes were kept below $5 \mathrm{k} \Omega$.

2.4. Behavioral Data Analysis. Reaction accuracy (RA) was the ratio of the number of correct responses to the total number of stimuli. Reaction time (RT) was measured from the onset of the stimulus to the time for response (pressing the " 1 " key) as indicated in the recordings. Any potential differences in RA and RT between the HCs and ADHD children were investigated by an independent $t$-test.

\subsection{EEG Data Analysis}

2.5.1. EEG Data Preprocessing. Multiple preprocessing procedures, including References Electrode Standardization Technique (REST) referencing [28-29], [1, 30] Hz bandpass filtering, 1-s-length segmenting $(200 \mathrm{~ms}$ before and $800 \mathrm{~ms}$ after targets onset, [-200, 800] ms) (0 ms denotes 


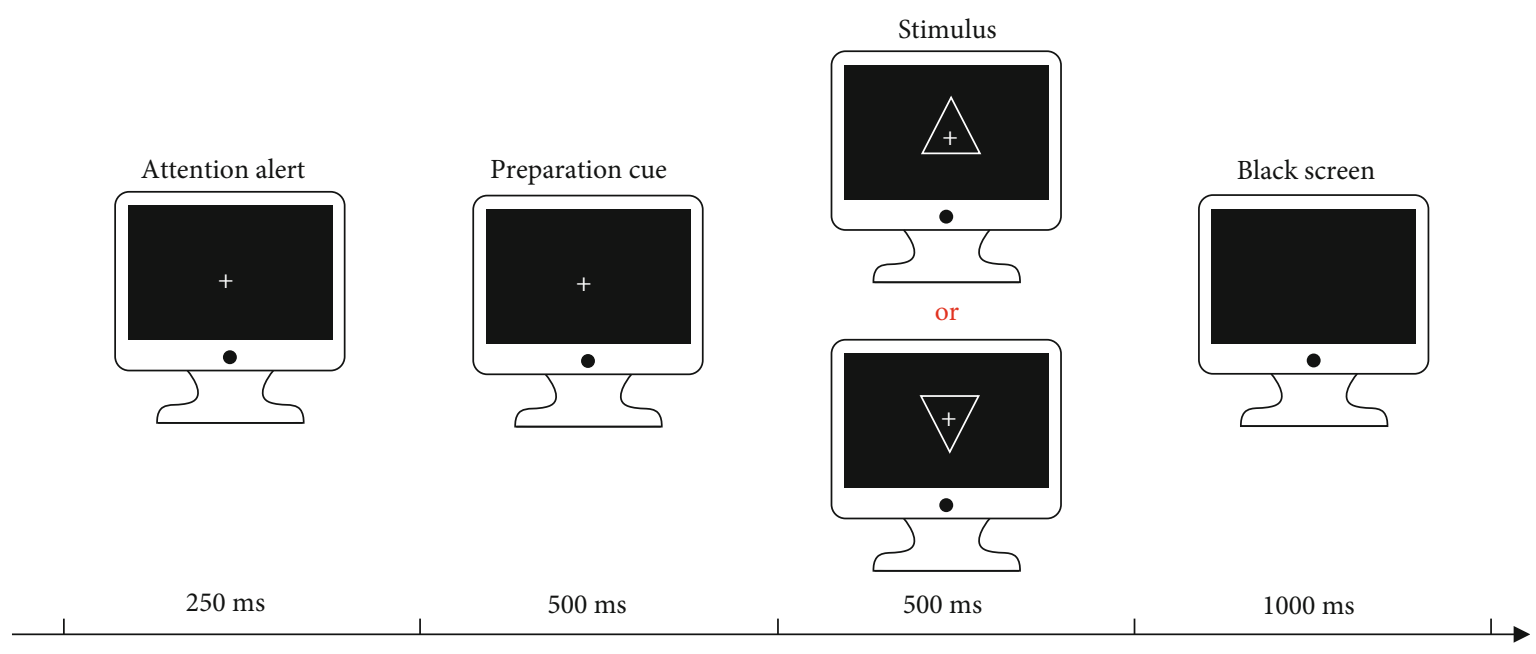

Trial $N$

Figure 1: Experimental protocol of the visual P3 tasks used in this study. In each trial, a $250 \mathrm{~ms}$ attention alert, a $500 \mathrm{~ms}$ preparation cue, a $500 \mathrm{~ms}$ stimulus presentation, and a $1000 \mathrm{~ms}$ break were included. The upward-oriented triangles and down-oriented triangles with a thin cross in the center denoted standard and target stimuli, respectively. Only standard or target stimulus appeared once in one trial.

stimuli onset), [-200, 0] ms baseline correction, and artifact-trial removal using a threshold of $\pm 100 \mu \mathrm{V}$ were implemented to preprocess the EEG datasets. In fact, to evoke the clear P3, subjects have to pay all of their attention to the target stimuli, by mentally counting the target number or physically pressing the required button [30], which was clarified as the maintained attention to the targets rather than the essence of P3 generation [31]. After carefully checking the evoked ERPs of these left trials, the remaining 25.45 \pm 2.78 and $26.84 \pm 1.75$ trials for HCs and ADHD children were included in the following analysis, respectively, which was consistently larger than the minimum trial number (i.e., 20) suggested previously [32].

2.5.2. ERP Components. After preprocessing, all artifact-free trials were trial-averaged for each subject. Then, according to previous studies $[7,33]$, the relatively broad intervals of $[150,200] \mathrm{ms}$ for P2, [200, 250] ms for N2, and [250, 600] ms for P3 were applied to the averaged ERP, to extract the corresponding P2, N2, and P3 amplitudes. Herein, the corresponding amplitude per ERP component was calculated as the mean amplitude in a $20 \mathrm{~ms}$ window centered at its peak. Thereafter, the differences in P2, N2, and P3 between the $\mathrm{HCs}$ and ADHD children were statistically investigated by an independent $t$-test.

2.5.3. Brain Network. The phase lock value (PLV) was proposed to estimate the phase synchronization among each pair of signals [34] and has been widely used in neuroscience research, such as ADHD [35]. The corresponding PLV value is in a range of $[0,1]$ with higher values representing the stronger rhythm locking.

To estimate the corresponding instantaneous phases, $\phi_{x}$ $(t)$ and $\phi_{y}(t)$, of two given signals, $x(t)$ and $y(t)$, the analytical signal $H(t)$ is defined by the Hilbert transform (HT) as
TABLE 1: Behavioral data.

\begin{tabular}{lccccc}
\hline & \multicolumn{2}{c}{ HCs } & \multicolumn{2}{c}{ ADHD children } & \multirow{2}{*}{ Mealue } \\
& Mean & SD & Mean & SD & \\
\hline RA (\%) & 65.7 & 21.1 & 58.3 & 22.7 & $p>0.05$ \\
RT (s) & 651.5 & 153.3 & 648.9 & 158.4 & $p>0.05$ \\
\hline
\end{tabular}

follows:

$$
\left\{\begin{array}{l}
H_{x}(t)=x(t)+i \widehat{x}(t), \\
H_{y}(t)=y(t)+i \widehat{y}(t),
\end{array}\right.
$$

where $\hat{x}(t)$ and $\hat{y}(t)$ are the HT of $x(t)$ and $y(t)$, which are defined as follows:

$$
\left\{\begin{array}{l}
\widehat{x}(t)=\frac{1}{\pi} \mathrm{P} . \mathrm{V} \cdot \int_{-\infty}^{\infty} \frac{x(\tau)}{t-\tau} d \tau \\
\widehat{y}(t)=\frac{1}{\pi} \mathrm{P} . \mathrm{V} \cdot \int_{-\infty}^{\infty} \frac{y(\tau)}{t-\tau} d \tau
\end{array}\right.
$$

where P.V. stands for the Cauchy principal value. Then, the corresponding instantaneous phases, $\phi_{x}(t)$ and $\phi_{y}(t)$, can be computed as follows:

$$
\left\{\begin{array}{l}
\phi_{x}(t)=\arctan \frac{\widehat{x}(t)}{x(t)} \\
\phi_{y}(t)=\arctan \frac{\widehat{y}(t)}{y(t)}
\end{array}\right.
$$


P3

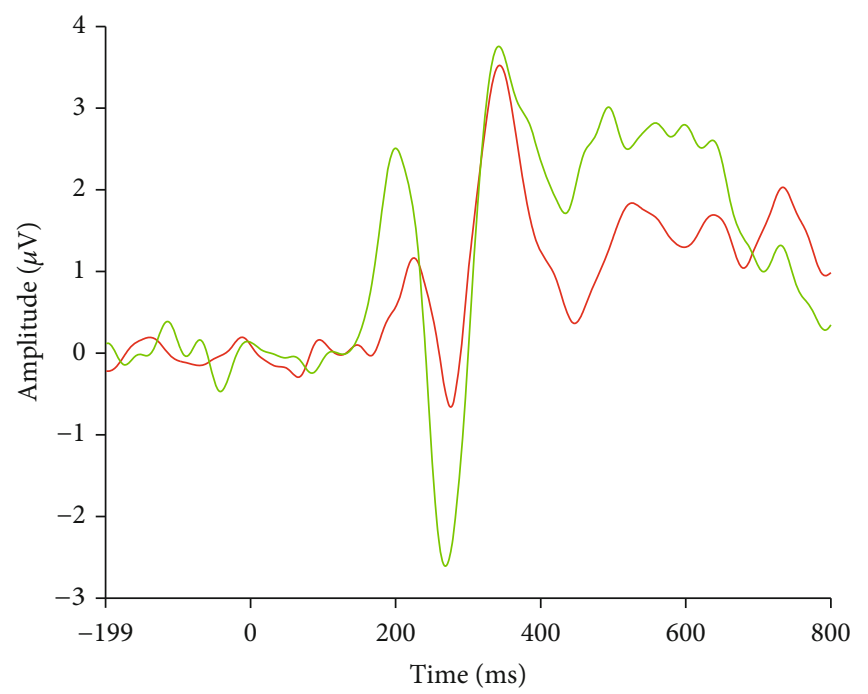

— ADHD children

- HCs

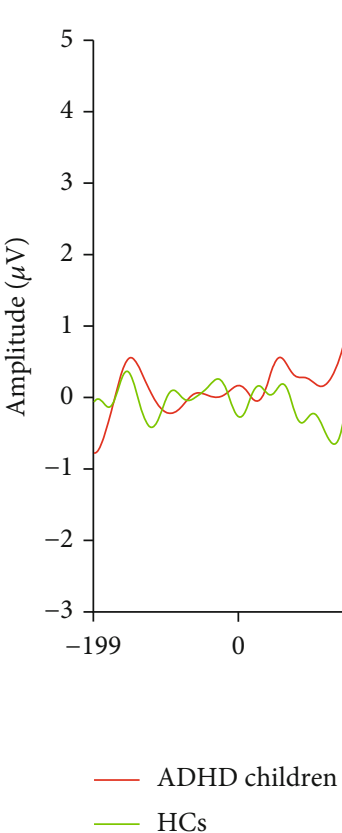

P4

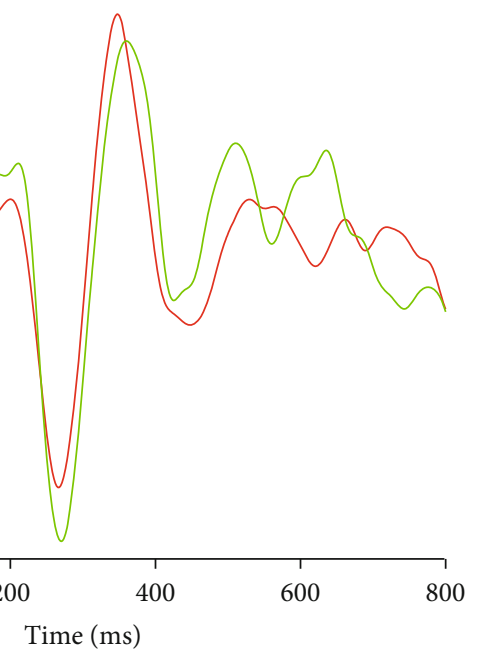

(b)

FIGURE 2: ERP waveforms at electrodes P3 (a) and P4 (b) between the HCs and ADHD children.

Finally, the PLV value can be estimated as follows:

$$
w^{\mathrm{plv}}=\frac{1}{N}\left|\sum_{n=0}^{N-1} e^{i\left(\phi_{x}(n \Delta t)-\phi_{y}(n \Delta t)\right)}\right|,
$$

where $w^{\text {plv }}$ is the connection weight estimated by PLV, $\Delta t$ is the sampling interval, and $N$ denotes the sample number.

Based on the PLV, the adjacency matrix per trial per subject was first calculated. Afterwards, for each subject, the final weighted network, a $16 \times 16$ adjacency matrix, was acquired by averaging matrices across all trials. Based on these constructed brain networks, an independent $t$-test was used to investigate the potential difference in brain architecture between the two groups.

2.5.4. Source Localization. In our present study, when estimating the cortical current density of P2 and N2, the sLORETA (v20171101) was adopted. The cortex has been modeled as a collection of volume elements (voxels) in the digitized atlas provided by the Brain Imaging Center, Montreal Neurological Institute (MNI) [36]. The sLORETA method found a particular solution to the nonunique EEG inverse problem by assuming similar activation of neighbouring neuronal sources, followed by an appropriate standardization of the current density, producing images of electric neuronal activity [37]. Regarding the technical details of the sLORETA procedure, the MNI brain volume was scanned at a resolution of $5 \mathrm{~mm}$. Voxels were retained when they were unambiguously labeled as cortical gray matter and when they were unambiguously within the brain compartment. sLORETA's solution space was therefore restricted to that cortical and hippocampal gray matter whose images represented the power in 6239 voxels, with a spatial resolution of $5 \mathrm{~mm}$. Anatomical labels were reported using an appropriate correction from MNI to Talairach space [38]. Besides, the lead field matrix was computed via a standardized boundary element method model volume conductor model with the realistic head model [39]. Herein, based on sLORETA, we estimated the current density of each ERP component for both HCs and ADHD children. The mean of sLORETA solutions corresponding to the ERP components was obtained within their time interval within which the ERP components were extracted. Then, the differences corresponding to the cortical activity between the two groups were statistically investigated by an independent $t$-test.

\section{Results}

3.1. Behavior. No significant differences in RA and RT between the two groups were first found in this study, as shown in Table 1.

3.2. ERP. Thereafter, when investigating the concerned ERP components on electrodes $\mathrm{P} 3$ and $\mathrm{P} 4$, similar results on both electrodes were found. In detail, no significant difference in P3 was found between the HCs and ADHD children. On the contrary, the amplitudes of both $\mathrm{P} 2$ and $\mathrm{N} 2$ were reduced in ADHD children, when compared to HCs $(p<0.05)$, as displayed in Figure 2.

Given the differences in N2 and P2, the differences corresponding to the cortical activity between the two groups were statistically investigated, which are shown in Figure 3. In detail, compared to ADHD children, HCs showed much stronger activity in frontal, cingulate, and central areas for P2 $(p<0.01)$ and in temporal, frontal, and occipital regions for N2 $(p<0.01)$, whose MNI coordinates, T-value, and voxel number are listed in Tables 2 and 3, respectively. 

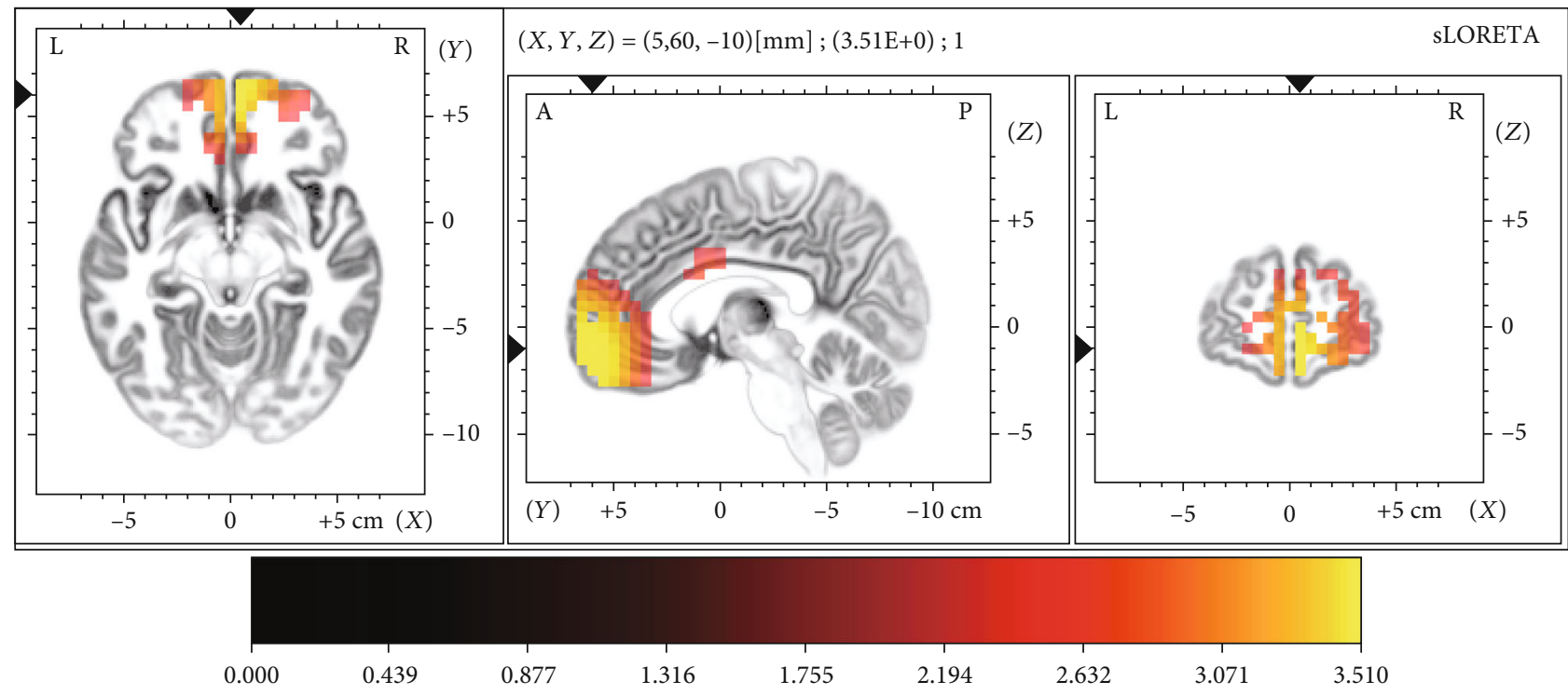

(a)
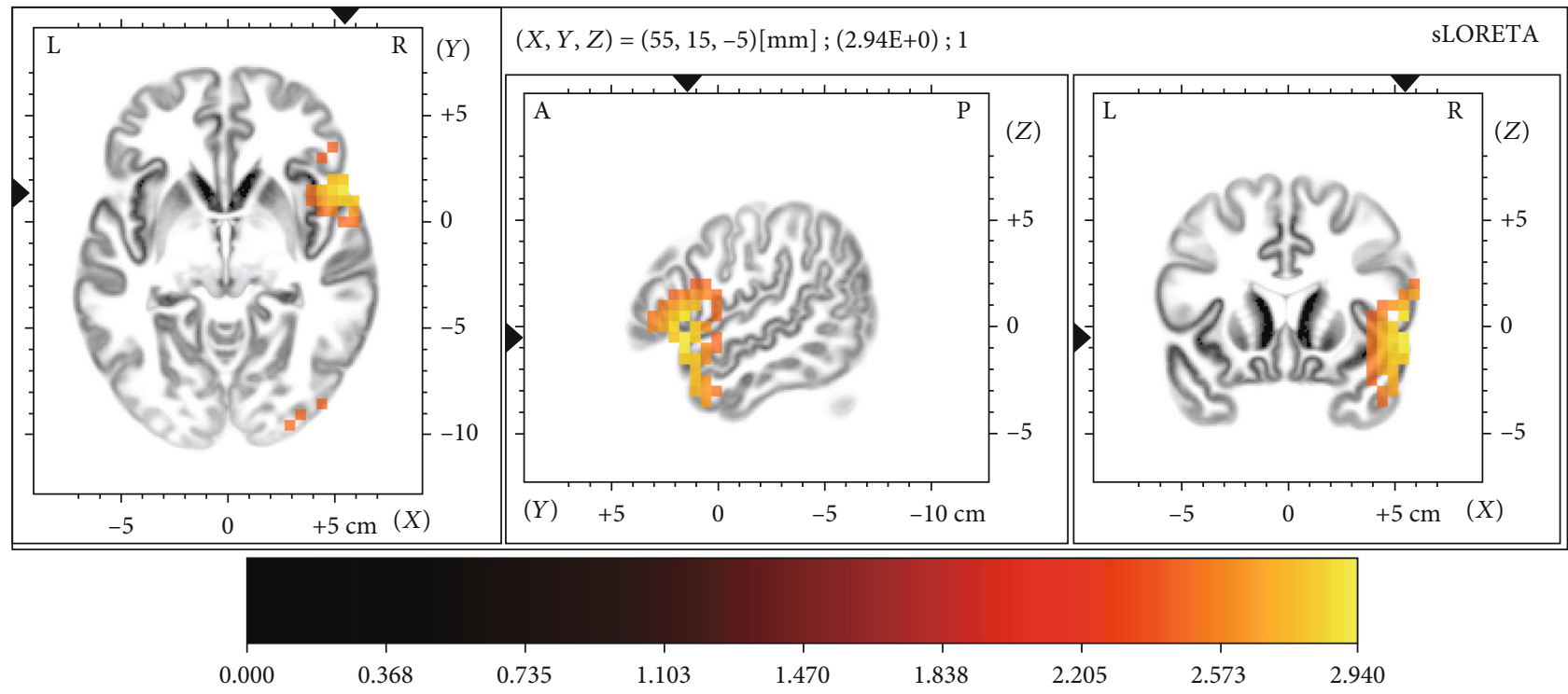

(b)

Figure 3: The T-value statistical map corresponding to the P2 and N2. (a) The stronger activity for P2 in HCs; (b) the stronger activity for N2 in HCs. The distinct colors presented the activity of the related brain regions in each subfigure.

TABLE 2: The details of the stronger activity for P2 in HCs.

\begin{tabular}{|c|c|c|c|c|c|c|c|}
\hline \multirow{2}{*}{ Activated region } & \multirow{2}{*}{$\mathrm{L} / \mathrm{R}$} & \multirow{2}{*}{$\mathrm{BA}$} & \multicolumn{3}{|c|}{ MNI coordinates } & \multirow{2}{*}{$T$-value } & \multirow{2}{*}{ Numbers of voxels } \\
\hline & & & $x$ & $y$ & $z$ & & \\
\hline Medial frontal gyrus & $\mathrm{L} / \mathrm{R}$ & $9,10,11,25$ & 5 & 60 & -10 & 3.51 & 111 \\
\hline Superior frontal gyrus & $\mathrm{L} / \mathrm{R}$ & 10,11 & 5 & 60 & -20 & 3.51 & 92 \\
\hline Anterior cingulate & $\mathrm{L} / \mathrm{R}$ & $10,24,32,33$ & 5 & 55 & 0 & 3.45 & 60 \\
\hline Postcentral gyrus & $\mathrm{L} / \mathrm{R}$ & $1,2,3,40$ & -50 & -20 & 60 & 2.49 & 30 \\
\hline Middle frontal gyrus & $\mathrm{L} / \mathrm{R}$ & 10,11 & 25 & 55 & -10 & 2.78 & 22 \\
\hline
\end{tabular}


TABLE 3: The details of the stronger activity for N2 in HCs.

\begin{tabular}{|c|c|c|c|c|c|c|c|}
\hline \multirow{2}{*}{ Activated region } & \multirow{2}{*}{$\mathrm{L} / \mathrm{R}$} & \multirow{2}{*}{ BA } & \multicolumn{3}{|c|}{ MNI coordinates } & \multirow{2}{*}{$T$-value } & \multirow{2}{*}{ Numbers of voxels } \\
\hline & & & $x$ & $y$ & $z$ & & \\
\hline Superior temporal gyrus & $\mathrm{R}$ & $21,22,38$ & 55 & 15 & -5 & 2.94 & 58 \\
\hline Inferior frontal gyrus & $\mathrm{R}$ & $13,44,45,47$ & 55 & 15 & 5 & 2.89 & 58 \\
\hline Middle temporal gyrus & $\mathrm{R}$ & $19,21,38$ & 55 & 10 & -25 & 2.79 & 36 \\
\hline Middle occipital gyrus & $\mathrm{R}$ & 18,19 & 40 & -90 & 5 & 2.53 & 25 \\
\hline
\end{tabular}

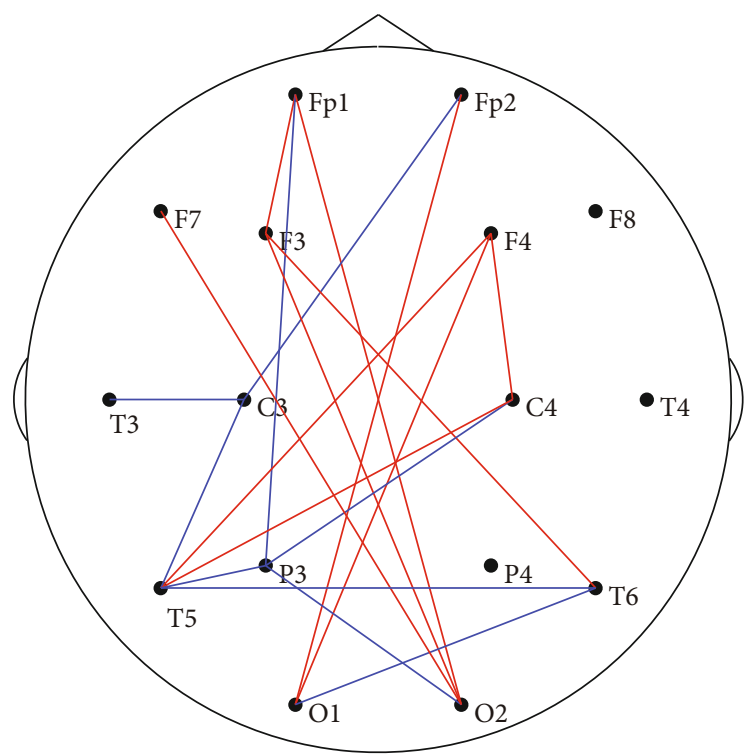

Figure 4: Differentiated brain topology between the HCs and ADHD children; the red and blue solid lines denote the stronger and weaker functional connectivity in ADHD children, respectively, when compared to HCs.

3.3. Brain Network. Figure 4 demonstrates the identified topological differences $(p<0.05)$ between the two groups. In detail, relatively stronger functional connectivity (i.e., red long-range edges) among frontal and occipital lobes but weaker linkages (i.e., blue edges) among frontal, parietal, and temporal regions were found for ADHD children, when compared to HCs.

\section{Discussion}

As previously illustrated, ADHD patients usually showed the task deficits in varied substage information processing, such as information integration and neuronal response stages, rather than the whole task period [5]. However, task behaviors usually measured the overall performance but not substage information processing [40], as behaviors were insensitive to detect the substage performance; by contrast, related ERP analysis might be helpful. Given the insignificant difference in behavior between the HCs and ADHD children, in this study, we further explored any potential difference in electrophysiological ERP and functional network to clarify underlying cognitive deficits in ADHD children.
Being exposed to external stimuli, to achieve satisfying task performance, the brain has to receive, integrate, process, and respond to their perceived target stimuli. P3 is the neuronal response to target stimuli after the decision process [41]. Li et al. [42] found that the central area served as the focal source to regulate whole-brain activities by sending out commands during the decision process, which ended when the P3 peak occurred. When investigating the underlying basis by the ERP, first at the response stage, our current study did not find a significant difference in P3 between the HCs and ADHD children, and this might indicate that the cognitive deficits between the two groups might not occur in the response stage but other ones, such as the information integration stage, which could be identified by the other components, like P2 and N2. In fact, in our present study, the corresponding electrophysiological differences in both P2 and N2 did exist between the two groups.

P2 is regarded as the exogenous response, as it is automatically produced regardless of the task or attention variables but is amenable to attentive manipulations, and its latency and amplitude may covary with aspects of selective attention or stimulus encoding processes [43]. Therefore, P2 involves in the early sensory stages of target detection [44], encoding, and classification [45]; a smaller P2 suggests an underactivation of the early orienting process and a poor allocation of the attentional resources in ADHD [46]. In this study, consistent with the previous study [47], ADHD children were found to have reduced P2 amplitude, which may be due to the lack of early attention to the stimuli. Moreover, $\mathrm{N} 2$ has been demonstrated to relate to the initial stimulus categorization in the selective attention stream [48] and was specific to the visual modality, which might reflect the degree of attention required for processing stimuli in the visual cortex [49]. Perhaps due to the lack of reasonable allocation of visual resources, attenuated N2 in ADHD children was found in this study. Altogether, these findings indicated that the differences in the cognitive process between the HCs and ADHD children may indeed lie in the early stage (i.e., information integration), rather than the later one (i.e., neuronal response). Therefore, ADHD children showed deficiency in automatization of the initial stimulus categorization for which they compensated by later controlled attention processes and information processing, which was earlier proposed by Karayanidis et al. [50].

More evidence underlying these differences would be acquired, when considering the cortical N2 and P2 activity. On the one hand, compared to ADHD children, HCs showed much stronger activity in frontal, cingulate, and central areas 
for P2. And these cortexes involved in the generation of P2 [51] and abnormalities in these regions were common in ADHD [52]. On the other hand, the temporal, especially superior temporal cortex, and frontal regions illustrated by the earlier study were both linked with N2 generators [53, 54]; in specific, the neuronal activity of frontotemporal regions was associated with $\mathrm{N} 2$ [55]. The reduced activity of the right temporal lobe in this study was consistent with that reported by Rubia et al. who found reduced activation in the right superior temporal lobe in ADHD patients during their attention tasks [56]. Besides, the occipital cortex contributes to attention processing [57] and has also been reported to be abnormal in ADHD [58].

Figure 4 further displays the atypical interactions underlying the task information processing for ADHD in which long-range connectivity between the frontal and occipital lobes could be found. In fact, during the visual task, the occipital lobe is responsible for receiving and integrating visual information, which was revealed by $\mathrm{Li}$ et al.'s study on constructing large-scale cortical networks for P3 [59], while the frontal lobe contributes to a wide range of cognitive functions, such as attention [60], decision-making [61], and executive control [62]. The frequency-specified synchronization between these regions can effectively modulate cognitive information processing [63]. And studies of both rats and humans have further consistently proved that long-range linkages between the frontal and occipital regions facilitate stronger visual evoked potentials [64, 65]. In addition, $\mathrm{Li}$ et al. found that the long-range frontal-occipital connectivity played crucial roles in P3 generation [66]. The enhanced frontal-occipital connectivity may alternatively compensate for the deficit in the early information integration stage, which thus facilitated their accomplishing the P3 task.

Given that only EEG datasets of several ADHD girls were collected, one possible limitation was that our present study did not take the gender effect into consideration; in the future, the gender-matched ADHD children will be recruited to validate if the gender could affect our present findings. Another might be that the task behaviors were not taken into consideration when selecting reliable task trials; in our future, more detailed individual behaviors would be recorded and then analyzed during preprocessing to further validate our current findings.

\section{Conclusion}

Although no significant differences in task behavior between the HCs and ADHD children were found, electrophysiological ERP and functional network did uncover the potential cognitive deficits in ADHD children, especially in the early task stage. In particular, significant differences in both N2 and P2 amplitudes and cortical activity, but not in P3, between the two groups were first found. And further, the topological differences showed the attenuated functional connectivity among frontal, parietal, and temporal lobes in ADHD children, which might be compensated by the enhanced long-range frontal-occipital connectivity to accomplish the required tasks.

\section{Data Availability}

The data used to support the findings of this study are available from the corresponding authors upon request.

\section{Conflicts of Interest}

The authors declare that there is no conflict of interest regarding the publication of this paper.

\section{Authors' Contributions}

Chunli Chen and Huan Yang equally contributed to the work.

\section{Acknowledgments}

This work was supported by the Key Research and Development Program of Guangdong Province, China (\#2018B030339001); the National Natural Science Foundation of China (\#61961160705, \#U19A2082, and \#61901077); the National Key Research and Development Plan of China (\#2017YFB1002501); the Science and Technology Development Fund, Macau SAR (File no. 0045/2019/AFJ); and the CAMS Innovation Fund for Medical Sciences (CIFMS) (No. 2019-I2M-5-039).

\section{References}

[1] A P Association, Diagnostic and Statistical Manual of Mental Disorders (DSM-5 $\left.{ }^{\circledR}\right)$, American Psychiatric Pub, 2013.

[2] J. T. Nigg and B. J. Casey, "An integrative theory of attentiondeficit/hyperactivity disorder based on the cognitive and affective neurosciences," Development and Psychopathology, vol. 17, no. 3, pp. 785-806, 2005.

[3] G. A. Stefanatos and I. S. Baron, "Attention-deficit/hyperactivity disorder: a neuropsychological perspective towards DSMV," Neuropsychology Review, vol. 17, no. 1, pp. 5-38, 2007.

[4] A. Lenartowicz and S. K. Loo, "Use of EEG to diagnose ADHD," Current Psychiatry Reports, vol. 16, no. 11, pp. 111, 2014.

[5] S. J. Johnstone, R. J. Barry, and A. R. Clarke, "Ten years on: A follow-up review of ERP research in attention- deficit/hyperactivity disorder," Clinical Neurophysiology, vol. 124, no. 4, pp. 644-657, 2013.

[6] G. E. Raney, "Monitoring changes in cognitive load during reading: an event-related brain potential and reaction time analysis," Journal of Experimental Psychology: Learning, Memory, and Cognition, vol. 19, no. 1, pp. 51-69, 1993.

[7] M. Senderecka, A. Grabowska, J. Szewczyk, K. Gerc, and R. Chmylak, "Response inhibition of children with ADHD in the stop-signal task: an event-related potential study," International Journal of Psychophysiology, vol. 85, no. 1, pp. 93-105, 2012.

[8] S. J. Johnstone, R. J. Barry, V. Markovska, A. Dimoska, and A. R. Clarke, "Response inhibition and interference control in children with AD/HD: a visual ERP investigation," International Journal of Psychophysiology, vol. 72, no. 2, pp. 145-153, 2009.

[9] M. Liotti, S. R. Pliszka, K. Higgins, R. P. Iii, and M. SemrudClikeman, "Evidence for specificity of ERP abnormalities 
during response inhibition in ADHD children: a comparison with reading disorder children without ADHD," Brain and Cognition, vol. 72, no. 2, pp. 228-237, 2010.

[10] R. J. Barry, S. J. Johnstone, and A. R. Clarke, “A review of electrophysiology in attention-deficit/hyperactivity disorder: II. Event-related potentials," Clinical Neurophysiology, vol. 114, no. 2, pp. 184-198, 2003.

[11] F. Li, J. Wang, Y. Liao et al., "Differentiation of schizophrenia by combining the spatial EEG brain network patterns of rest and task P300," IEEE Transactions on Neural Systems and Rehabilitation Engineering, vol. 27, no. 4, pp. 594-602, 2019.

[12] A. Kaiser, P.-M. Aggensteiner, S. Baumeister, N. E. Holz, T. Banaschewski, and D. Brandeis, "Earlier versus later cognitive event-related potentials (ERPs) in attention-deficit/hyperactivity disorder (ADHD): a meta-analysis," Neuroscience \& Biobehavioral Reviews, vol. 112, pp. 117-134, 2020.

[13] P. D. Gajewski, P. Stoerig, and M. Falkenstein, "ERP-correlates of response selection in a response conflict paradigm," Brain Research, vol. 1189, pp. 127-134, 2008.

[14] E. Bullmore and O. Sporns, "Complex brain networks: graph theoretical analysis of structural and functional systems," Nature Reviews Neuroscience, vol. 10, no. 3, pp. 186-198, 2009.

[15] F. Li, W. Peng, Y. Jiang et al., "The dynamic brain networks of motor imagery: time-varying causality analysis of scalp EEG," International Journal of Neural Systems, vol. 29, no. 1, article 1850016, 2019.

[16] Y. Si, X. Wu, F. Li et al., "Different decision-making responses occupy different brain networks for information processing: a study based on EEG and TMS," Cerebral Cortex, vol. 29, no. 10, pp. 4119-4129, 2019.

[17] F. Li, Q. Tao, W. Peng et al., "Inter-subject P300 variability relates to the efficiency of brain networks reconfigured from resting- to task-state: Evidence from a simultaneous eventrelated EEG-fMRI study," NeuroImage, vol. 205, p. 116285, 2020.

[18] D. J. Englot, L. B. Hinkley, N. S. Kort et al., "Global and regional functional connectivity maps of neural oscillations in focal epilepsy," Brain, vol. 138, no. 8, pp. 2249-2262, 2015.

[19] P. Lin, J. Sun, G. Yu et al., "Global and local brain network reorganization in attention-deficit/hyperactivity disorder," Brain Imaging and Behavior, vol. 8, no. 4, pp. 558-569, 2014.

[20] K. Konrad and S. B. Eickhoff, "Is the ADHD brain wired differently? A review on structural and functional connectivity in attention deficit hyperactivity disorder," Human Brain Mapping, vol. 31, no. 6, pp. 904-916, 2010.

[21] E. M. Valera, S. V. Faraone, K. E. Murray, and L. J. Seidman, "Meta-analysis of structural imaging findings in attentiondeficit/hyperactivity disorder," Biological Psychiatry, vol. 61, no. 12, pp. 1361-1369, 2007.

[22] D. E. Hill, R. A. Yeo, R. A. Campbell, B. Hart, J. Vigil, and W. Brooks, "Magnetic resonance imaging correlates of attention-deficit/hyperactivity disorder in children," Neuropsychology, vol. 17, no. 3, pp. 496-506, 2003.

[23] P. Shaw, K. Eckstrand, W. Sharp et al., "Attention-deficit/hyperactivity disorder is characterized by a delay in cortical maturation," Proceedings of the National Academy of ences, vol. 104, no. 49, pp. 19649-19654, 2007.

[24] D. S. Bassett and O. Sporns, "Network neuroscience," Nature Neuroscience, vol. 20, no. 3, pp. 353-364, 2017.

[25] G. Bush, E. M. Valera, and L. J. Seidman, "Functional neuroimaging of attention-deficit/hyperactivity disorder: a review and suggested future directions," Biological Psychiatry, vol. 57, no. 11, pp. 1273-1284, 2005.

[26] S. Furlong, J. R. Cohen, J. Hopfinger, J. Snyder, M. M. Robertson, and M. A. Sheridan, "Resting-state EEG connectivity in young children with ADHD," Journal of Clinical Child \& Adolescent Psychology, pp. 1-17, 2020.

[27] S. R. Srinivasan, "Functional connectivity of frontal cortex in healthy and ADHD children reflected in EEG coherence," Cerebral Cortex, vol. 17, no. 8, pp. 1788-1799, 2007.

[28] D. Yao, "Is the surface potential integral of a dipole in a volume conductor always zero? A cloud over the average reference of EEG and ERP," Brain Topography, vol. 30, no. 2, pp. 161171, 2017.

[29] D. Yao, "A method to standardize a reference of scalp EEG recordings to a point at infinity," Physiological Measurement, vol. 22, no. 4, pp. 693-711, 2001.

[30] R. Mertens and J. Polich, "P300 from a single-stimulus paradigm: passive versus active tasks and stimulus modality," Electroencephalography and Clinical Neurophysiology/Evoked Potentials Section, vol. 104, no. 6, pp. 488-497, 1997.

[31] E. H. Meijer, F. T. Y. Smulders, and A. Wolf, "The contribution of mere recognition to the P300 effect in a concealed information test," Applied Psychophysiology and Biofeedback, vol. 34, no. 3, pp. 221-226, 2009.

[32] E. Ghumare, M. Schrooten, R. Vandenberghe, and P. Dupont, "Comparison of different Kalman filter approaches in deriving time varying connectivity from EEG data," Annual International Conference of the IEEE Engineering in Medicine and Biology Society, vol. 2015, pp. 2199-2202, 2015.

[33] S. Ludyga, S. Brand, M. Gerber et al., "An event-related potential investigation of the acute effects of aerobic and coordinative exercise on inhibitory control in children with ADHD," Developmental Cognitive Neuroscience, vol. 28, pp. 21-28, 2017.

[34] J. Sun, Z. Li, and S. Tong, "Inferring functional neural connectivity with phase synchronization analysis: a review of methodology," Computational and Mathematical Methods in Medicine, vol. 2012, 13 pages, 2012.

[35] E. Pereda, M. García-Torres, B. Melián-Batista, S. Mañas, L. Méndez, and J. J. González, "The blessing of dimensionality: feature selection outperforms functional connectivity-based feature transformation to classify ADHD subjects from EEG patterns of phase synchronisation," PLoS One, vol. 13, no. 8, article e0201660, 2018.

[36] J. Talairach, “3-dimensional proportional system; an approach to cerebral imaging. co-planar stereotaxic atlas of the human brain," Thieme, pp. 1-122, 1988.

[37] R. D. Pascual-Marqui, "Standardized low-resolution brain electromagnetic tomography (sLORETA): technical details," Methods and Findings in Experimental and Clinical Pharmacology, vol. 24, pp. 5-12, 2002.

[38] M. Brett, I. S. Johnsrude, and A. M. Owen, "The problem of functional localization in the human brain," Nature Reviews Neuroscience, vol. 3, no. 3, pp. 243-249, 2002.

[39] M. Fuchs, J. Kastner, M. Wagner, S. Hawes, and J. S. Ebersole, "A standardized boundary element method volume conductor model," Clinical Neurophysiology, vol. 113, no. 5, pp. 702-712, 2002.

[40] M. R. Harter, L. Anllo-Vento, F. B. Wood, and M. M. Schroeder, "II. Separate brain potential characteristics in children with reading disability and attention deficit disorder: color 
and letter relevance effects," Brain and Cognition, vol. 7, no. 1, pp. 115-140, 1988.

[41] T. W. Picton, "The P300 wave of the human event-related potential," Journal of Clinical Neurophysiology, vol. 9, no. 4, pp. 456-479, 1992.

[42] F. Li, B. Chen, H. Li et al., "The time-varying networks in P300:a task-evoked EEG study," IEEE Transactions on Neural Systems and Rehabilitation Engineering, vol. 24, no. 7, pp. 725733, 2016.

[43] K. E. Crowley and I. M. Colrain, "A review of the evidence for P2 being an independent component process: age, sleep and modality," Clinical Neurophysiology, vol. 115, no. 4, pp. 732744, 2004.

[44] S. J. Luck and S. A. Hillyard, "Electrophysiological correlates of feature analysis during visual search," Psychophysiology, vol. 31, no. 3, pp. 291-308, 1994.

[45] B. R. Dunn, D. A. Dunn, M. Languis, and D. Andrews, "The relation of ERP components to complex memory processing," Brain and Cognition, vol. 36, no. 3, pp. 355-376, 1998.

[46] L. Jonkman, C. Kemner, M. Verbaten et al., “Attentional capacity, a probe ERP study: differences between children with attention-deficit hyperactivity disorder and normal control children and effects of methylphenidate," Psychophysiology, vol. 37, no. 3, pp. 334-346, 2000.

[47] C. R. Brown, A. R. Clarke, R. J. Barry, R. McCarthy, M. Selikowitz, and C. Magee, "Event-related potentials in attention-deficit/hyperactivity disorder of the predominantly inattentive type: an investigation of EEG-defined subtypes," International Journal of Psychophysiology, vol. 58, no. 1, pp. 94-107, 2005.

[48] V. D. Calhoun, L. Wu, K. A. Kiehl, T. Eichele, and G. D. Pearlson, "Aberrant processing of deviant stimuli in schizophrenia revealed by fusion of fMRI and EEG data," Acta neuropsychiatrica, vol. 22, no. 3, pp. 127-138, 2010.

[49] S. Suwazono, L. Machado, and R. T. Knight, "Predictive value of novel stimuli modifies visual event-related potentials and behavior," Clinical Neurophysiology, vol. 111, no. 1, pp. 2939, 2000 .

[50] F. Karayanidis, P. Robaey, M. Bourassa, D. de Koning, G. Geoffroy, and G. Pelletier, "ERP differences in visual attention processing between attention-deficit hyperactivity disorder and control boys in the absence of performance differences," Psychophysiology, vol. 37, no. 3, pp. 319-333, 2000.

[51] L. Zhou, G. Wang, C. Nan, H. Wang, Z. Liu, and H. Bai, "Abnormalities in P300 components in depression: an ERPsLORETA study," Nordic Journal of Psychiatry, vol. 73, no. 1, pp. 1-8, 2019.

[52] C. Tang, Y. Wei, J. Zhao, and J. Nie, “Different developmental pattern of brain activities in ADHD: a study of resting-state fMRI," Developmental Neuroscience, vol. 40, no. 3, pp. 246257, 2018.

[53] M. Downes, J. Bathelt, and M. D. Haan, "Event-related potential measures of executive functioning from preschool to adolescence," Developmental Medicine and Child Neurology, vol. 59, no. 6, pp. 581-590, 2017.

[54] R. Huster, R. Westerhausen, C. Pantev, and C. Konrad, "The role of the cingulate cortex as neural generator of the N200 and P300 in a tactile response inhibition task," Human Brain Mapping, vol. 31, no. 8, pp. 1260-1271, 2010.
[55] J. Kayser, G. E. Bruder, C. E. Tenke, B. K. Stuart, X. F. Amador, and J. M. Gorman, "Event-related brain potentials (ERPs) in schizophrenia for tonal and phonetic oddball tasks," Biological Psychiatry, vol. 49, no. 10, pp. 832-847, 2001.

[56] K. Rubia, A. B. Smith, M. J. Brammer, and E. Taylor, "Temporal lobe dysfunction in medication-naïve boys with attentiondeficit/hyperactivity disorder during attention allocation and its relation to response variability," Biological Psychiatry, vol. 62, no. 9, pp. 999-1006, 2007.

[57] M. Oldehinkel, W. Francx, C. F. Beckmann, J. K. Buitelaar, and M. Mennes, "Resting state FMRI research in child psychiatric disorders," European Child \& Adolescent Psychiatry, vol. 22, no. 12, pp. 757-770, 2013.

[58] D. Wang, R. Hu, Q. Wang, and Q. Zhang, "Spatiotemporal consistency analysis of attention-deficit/hyperactivity disorder children," Neuroence Letters, vol. 734, p. 135099, 2020.

[59] F. Li, C. Yi, Y. Jiang et al., "The construction of large-scale cortical networks for P300 from scalp EEG," IEEE Access, vol. 6, pp. 68498-68506, 2018.

[60] S. W. Michalka, L. Kong, M. L. Rosen, B. G. Shinn-Cunningham, and D. C. Somers, "Short-term memory for space and time flexibly recruit complementary sensory-biased frontal lobe attention networks," Neuron, vol. 87, no. 4, pp. 882-892, 2015.

[61] Y. Si, L. Jiang, Q. Tao et al., "Predicting individual decisionmaking responses based on the functional connectivity of resting-state EEG," Journal of Neural Engineering, vol. 16, no. 6, article 066025, 2019.

[62] C. Anne, K. Etienne, and J. P. O'Doherty, "Reasoning, learning, and creativity: frontal lobe function and human decision-making," PLoS Biology, vol. 10, no. 3, article e1001293, 2012.

[63] M. Siegel, T. H. Donner, R. Oostenveld, P. Fries, and A. K. Engel, "Neuronal synchronization along the dorsal visual pathway reflects the focus of spatial attention," Neuron, vol. 60, no. 4, pp. 709-719, 2008.

[64] P. Xu, C. Tian, Y. Zhang et al., "Cortical network properties revealed by SSVEP in anesthetized rats," Scientific Reports, vol. 3, no. 1, pp. 2496-2496, 2013.

[65] Y. Zhang, P. Xu, Y. Huang, K. Cheng, and D. Yao, “SSVEP response is related to functional brain network topology entrained by the flickering stimulus," PLoS One, vol. 8, no. 9, 2013.

[66] F. Li, T. Liu, F. Wang et al., "Relationships between the restingstate network and the P3: evidence from a scalp EEG study," Scientific Reports, vol. 5, no. 1, pp. 15129-15129, 2015. 